\title{
Photoacoustic imaging of single circulating melanoma cells in vivo
}

Lidai Wang, Junjie Yao, Ruiying Zhang, Song Xu, Guo $\mathrm{Li}$, et al.

Lidai Wang, Junjie Yao, Ruiying Zhang, Song Xu, Guo Li, Jun Zou, Lihong $\mathrm{V}$. Wang, "Photoacoustic imaging of single circulating melanoma cells in vivo," Proc. SPIE 9323, Photons Plus Ultrasound: Imaging and Sensing 2015, 93230A (11 March 2015); doi: 10.1117/12.2076987

SPIE. Event: SPIE BiOS, 2015, San Francisco, California, United States 


\title{
Photoacoustic imaging of single circulating melanoma cells in vivo
}

\author{
Lidai Wang ${ }^{1}$, Junjie Yao ${ }^{1}$, Ruiying Zhang ${ }^{1}$, Song $\mathrm{Xu}^{2}$, Guo $\mathrm{Li}^{1}$, Jun Zou ${ }^{2}$, Lihong V. Wang ${ }^{1}$ * \\ ${ }^{1}$ Optical Imaging Laboratory, Department of Biomedical Engineering, Washington University in St. \\ Louis. Campus Box 1097, One Brookings Drive, St. Louis, Missouri 63130-4899, USA \\ ${ }^{2}$ Electrical \& Computer Engineering, Texas A\&M University \\ 3128 TAMU, College Station, TX 77843-3128, USA
}

\begin{abstract}
Melanoma, one of the most common types of skin cancer, has a high mortality rate, mainly due to a high propensity for tumor metastasis. The presence of circulating tumor cells (CTCs) is a potential predictor for metastasis. Label-free imaging of single circulating melanoma cells in vivo provides rich information on tumor progress. Here we present photoacoustic microscopy of single melanoma cells in living animals. We used a fast-scanning optical-resolution photoacoustic microscope to image the microvasculature in mouse ears. The imaging system has sub-cellular spatial resolution and works in reflection mode. A fast-scanning mirror allows the system to acquire fast volumetric images over a large field of view. A $500-\mathrm{kHz}$ pulsed laser was used to image blood and CTCs. Single circulating melanoma cells were imaged in both capillaries and trunk vessels in living animals. These high-resolution images may be used in early detection of CTCs with potentially high sensitivity. In addition, this technique enables in vivo study of tumor cell extravasation from a primary tumor, which addresses an urgent pre-clinical need.
\end{abstract}

Keywords: Photoacoustic imaging, circulating tumor cell, melanoma

\section{INTRODUCTION}

Melanoma is one of the most common types of skin cancer. As in many other types of cancer, the major challenge in treating melanoma is tumor metastasis, which is also one of the key reasons that this type of cancer has a high mortality rate. Circulating tumor cells (CTC) is one of the key predictors for tumor metastasis(1). In vivo imaging of rare CTCs may help early diagnosis of tumor metastasis and provides a powerful tool to study the fundamental mechanism of tumor cell extravasation from the primary tumor.

Optical-resolution photoacoustic microscopy (OR-PAM) offers high spatial resolution, optical absorption contrasts, and functional imaging capabilities (2-5). In addition, for melanoma cells, photoacoustic (PA) techniques can provide label-free imaging with high sensitivity. All of these advantages make OR-PAM an ideal candidate to image circulating melanoma cells.

Photons Plus Ultrasound: Imaging and Sensing 2015, edited by Alexander A. Oraevsky, Lihong V. Wang Proc. of SPIE Vol. 9323, 93230A - (c) 2015 SPIE - CCC code: 1605-7422/15/\$18 doi: $10.1117 / 12.2076987$ 
Here, we developed video-rate OR-PAM (6) for imaging of rare circulating melanoma cells in vivo. The imaging system has sub-cellular spatial resolution and works in reflection mode. A fastscanning mirror allows the system to acquire fast volumetric images over a large field of view. A $500-\mathrm{kHz}$ pulsed laser was used to image blood and CTCs. Single circulating melanoma cells were imaged in both capillaries and trunk vessels in living animals. These high-resolution images may be used in early detection of CTCs with potentially high sensitivity. In addition, this technique enables an in vivo study of tumor cell extravasation from a primary tumor, which addresses an urgent preclinical need.

\section{IMAGING SYSTEM DESIGN}

A fast pulsed laser (wavelength: $532 \mathrm{~nm}$ and $1064 \mathrm{~nm}$, pulse width: $3 \mathrm{ps}, 500 \mathrm{kHz}$ ) delivers light to the OR-PAM system(6). As shown in Figure 1, the laser beam is first focused by an optical objective, and then passes through an aberration-correction lens, and is reflected by a prism mirror. The reflected optical beam is further redirected onto the sample via a scanning mirror. The emitted spherical ultrasonic wave is firstly reflected by the same scanning mirror, and then collected by a concave acoustic lens. The acoustic lens converts the spherical ultrasonic wave into a planar wave, which passes through an optical/acoustic beam combiner, and is detected by a broadband ultrasonic transducer. The optical/acoustic beam combiner is made of two right-angle glass prisms with a thin layer of aluminum coating in the middle. Because aluminum coating has an acoustic impedance similar to that of glass, the beam combiner effectively transmits the ultrasonic wave and reflects the optical beam. The optical beam is precisely aligned with the ultrasonic beam, so that the imaging system can achieve high sensitivity. The scanning mirror can provide a one-dimensional (along the $x$-axis) fast mechanical scan in water, which maintains the alignment between the optical and acoustic beams. Another linear translational stage scans the animal in the elevational direction $(y$ axis). The imaging system provides fast imaging ability and high spatial resolution, which allows the detection of melanoma cells in vivo.

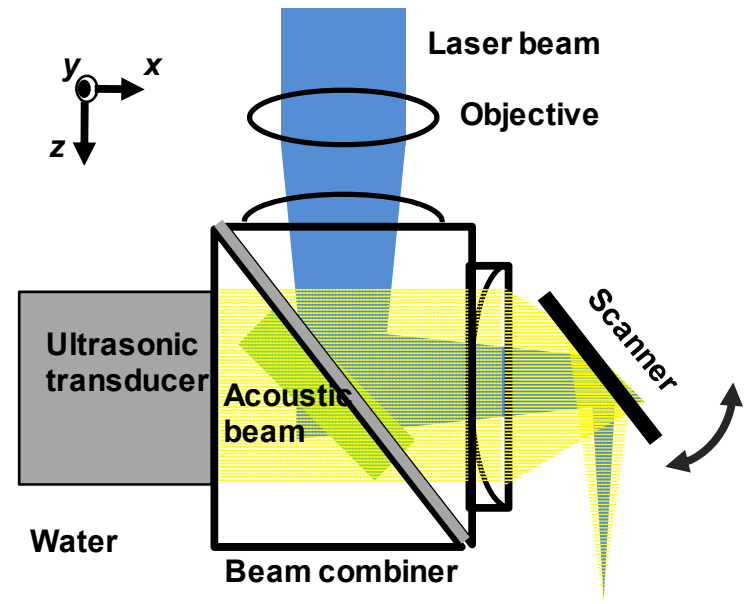

Figure 1. schematics of the fast-scanning OR-PAM system. 


\section{EXPERIMENTAL RESULTS}

In order to validate the system performance, we first imaged a blood phantom mixed with melanoma cells in a rubber tube. Using $532 \mathrm{~nm}$ wavelength, melanoma cells generated 200\% 300\% stronger photoacoustic signal than blood. With fast imaging ability and optically-defined lateral resolution, we can image single melanoma cells flowing with the blood.

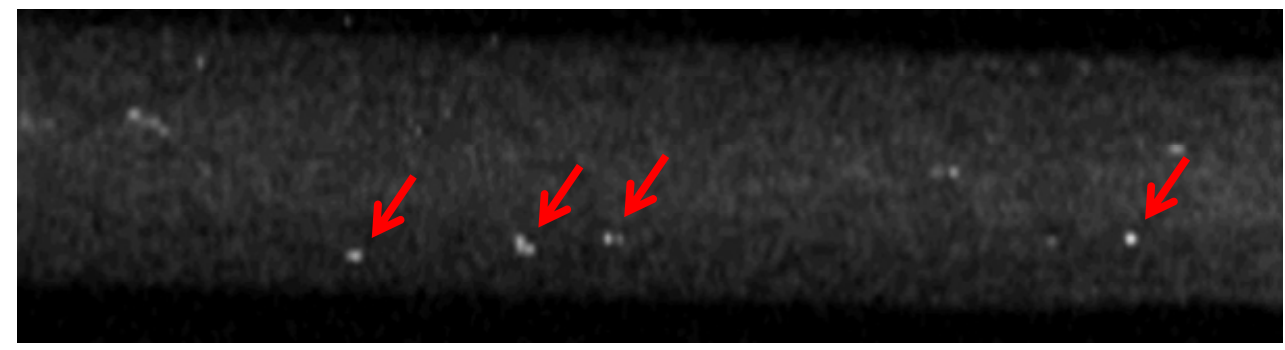

Figure 2. Imaging of melanoma cells in whole blood. Arrows point to melanoma cells

By injecting melanoma cells into the jugular vein of the mouse, we imaged circulating tumor cells in vivo. Figure 3 shows a melanoma cell passing a capillary vessel. The signal from the single melanoma cell is several times stronger than that from single red blood cells in the capillary. With fast imaging speed and high spatial resolution, we can also image single melanoma cells in bigger vessels. Figure 4 shows imaging of a circulating melanoma cell when it flows in a vein vessel. Control experiments had been carried out to validate that the strong signals were from melanoma cells.
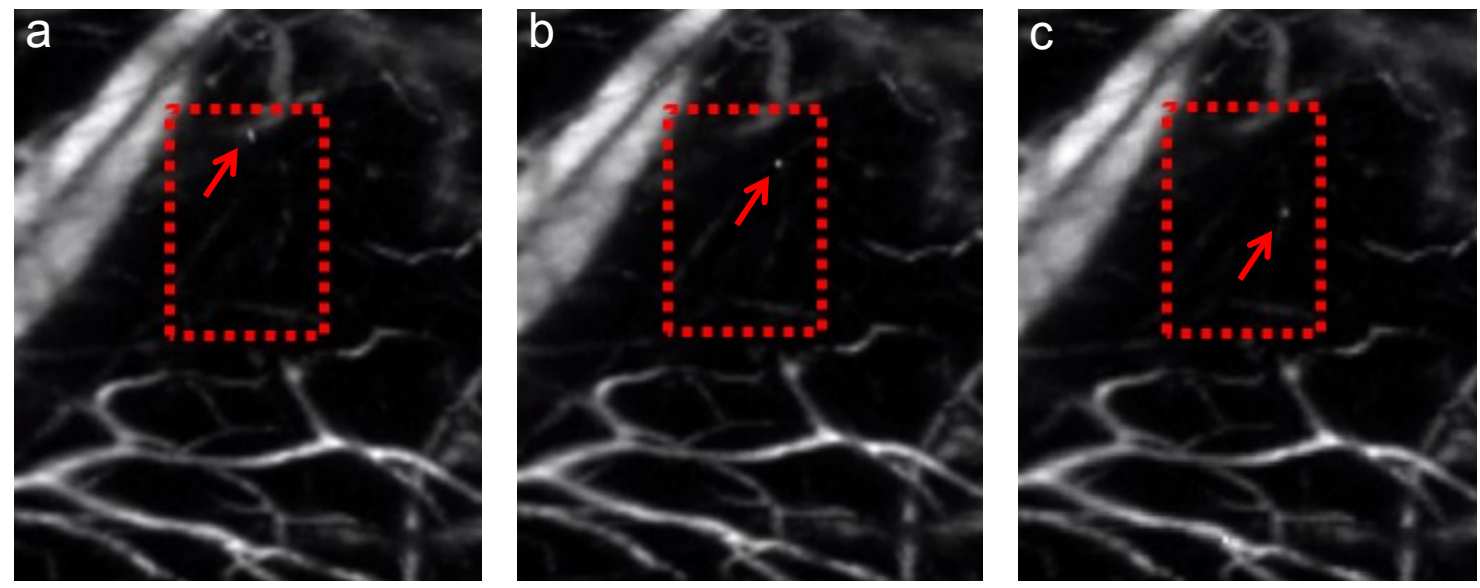

Figure 3. In vivo imaging of melanoma cell in capillary. Time interval between consecutive images is 1 seconds. Arrow pointing a melanoma cell passing through a capillary. 

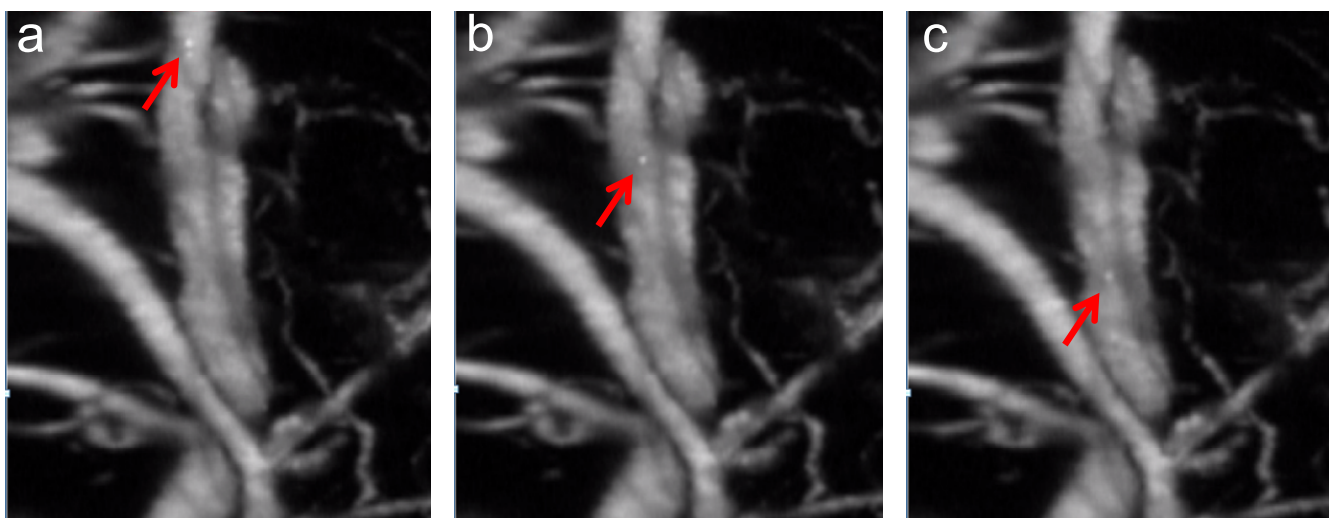

Figure 4. In vivo imaging of melanoma cell in vein. Time interval between consecutive images is 0.5 seconds. Arrow pointing a melanoma cell flow in a vein vessel.

\section{CONCLUSION}

In summary, we have successfully developed video-rate OR-PAM for imaging of circulating melanoma cells in vivo. CTCs in both capillary and trunk vessels have been imaged in real time. Our system even allows us to image CTCs with near-infrared wavelength in the future, which should provide higher imaging contrast. This method may be used in early detection of CTCs with potentially high sensitivity and in vivo study of tumor metastasis mechanism.

\section{REFERENCES}

[1] 1.L. Khoja, P. Lorigan, C. Dive, U. Keilholz and A. Fusi, "Circulating tumor cells as tumor biomarkers in melanoma: detection methods and clinical relevance," Annals of Oncology (2014)

[2] 2. E. I. Galanzha, E. V. Shashkov, P. M. Spring, J. Y. Suen and V. P. Zharov, "In vivo, Noninvasive, Label-Free Detection and Eradication of Circulating Metastatic Melanoma Cells Using Two-Color Photoacoustic Flow Cytometry with a Diode Laser," Cancer Research 69(20), 7926-7934 (2009)

[3] 3.L. Wang, K. Maslov, J. Yao, B. Rao and L. V. Wang, "Fast voice-coil scanning optical-resolution photoacoustic microscopy," Optics Letters 36(2), 139-141 (2011)

[4] 4.L. Wang, K. Maslov and L. V. Wang, "Single-cell label-free photoacoustic flowoxigraphy in vivo," Proceedings of the National Academy of Sciences 110(15), 5759-5764 (2013)

[5] 5.L. Wang, K. Maslov, W. Xing, A. Garcia-Uribe and L. V. Wang, "Video-rate functional photoacoustic microscopy at depths," Journal of Biomedical Optics 17(10), 106007-106001 (2012)

[6] 6. J. Yao, C.-H. Huang, L. Wang, J.-M. Yang, L. Gao, K. I. Maslov, J. Zou and L. V. Wang, "Wide-field fastscanning photoacoustic microscopy based on a water-immersible MEMS scanning mirror," Journal of Biomedical Optics 17(8), 080505 (2012) 